\title{
Use of Multidetector Row CT to Evaluate the Need for Bronchial Arterial Embolization in Hemoptysis Patients
}

\author{
Hidenori Moria Yasushi Ohno $^{a} \quad$ Yusuke Tsuge $^{\mathrm{b}} \quad$ Masanori Kawasaki $^{\mathrm{a}}$ \\ Fumitaka Ito $^{a}$ Junki Endo ${ }^{a}$ Norihiko Funaguchi ${ }^{a}$ Bu Lin Bai La ${ }^{a}$ \\ Masayuki Kanematsu $^{\mathrm{b}}$ Shinya Minatoguchi ${ }^{\mathrm{a}}$ \\ ${ }^{a}$ Second Department of Internal Medicine, and ${ }^{b}$ Department of Radiology, Gifu University Graduate School of \\ Medicine, Gifu, Japan
}

\section{Key Words}

Arteriography $\cdot$ Bronchial arteries $\cdot$ Bronchoscopy •

Computed tomography $\cdot$ Embolization $\cdot$ Hemoptysis

\begin{abstract}
Background: Bronchial artery (BA) embolization (BAE) is recommended as a minimally invasive therapy for hemoptysis, though some patients recover after only conservative treatment. Objectives: The purpose of our study was to assess the characteristics of BAs using multidetector row computed tomography (MDCT) and identify BAs requiring BAE without BA angiography (BAG). Methods: We retrospectively studied 41 patients and classified the visualized BAs into groups based on their BAE and bleeding statuses. Patients presenting with massive hemoptysis requiring emergency BAE were excluded. Patients presenting with persistent hemoptysis that was resistant to conservative treatment received BAE. Radiologists measured BA diameters at the ostium, bronchial bifurcation and pulmonary hilum, and also evaluated the degree of vascularization. Results: MDCT enabled visualization of 102 ostia and 96 traceable BAs. Among the participating patients, 13 had at least one ectopic origin. We obtained a good correlation between BAG and MDCT diameters $(r=0.709, p<0.001)$. The diameters of $B A s$ responsible for bleeding and receiving BAE were apparently larger
\end{abstract}

in each measured segment than those that were not $(p<$ 0.05). Moreover, the diameters of arteries receiving BAE remained largely unchanged from the origin to the hilum and through the mediastinum. BAs with low MDCT scores were significantly less likely to required $B A E$ than those with high scores $(p=0.004)$, and in multiple logistic regression analysis, ostium diameter and bleeding status were independent predictive factors for BAE. Conclusions: Evaluation of BAs on MDCT could be useful for identifying the anatomical characteristics of bleeding-related BAs and determining whether $B A E$ is indicated or whether conservative treatment is sufficient.

Copyright $\odot 2009$ S. Karger AG, Basel

\section{Introduction}

Hemoptysis is a common but alarming and potentially life-threatening symptom defined as expectoration of blood from the lower respiratory tract [1]. Emergency surgery in patients with active bleeding is associated with a high degree of morbidity and mortality, and bronchial artery (BA) embolization (BAE) is recommended for particularly high-risk patients [2-4]. But BAE occasionally causes a serious complication such as cerebral infarction or spinal cord injury [5]; moreover, some hemoptysis pa-

\section{KARGER}

Fax +4161306 1234

E-Mail karger@karger.ch

www.karger.com (c) $2009 \mathrm{~S}$. Karger AG, Basel

0025-7931/10/0801-0024\$26.00/0

Accessible online at:

www.karger.com/res
Shinya Minatoguchi, MD

Second Department of Internal Medicine

Gifu University Graduate School of Medicine

Yanagido 1-1, Gifu City, Gifu 501-1194 (Japan)

Tel. +81 58230 6523, Fax +81 582306524 , E-Mail minatos@gifu-u.ac.jp 
tients recover with only conservative treatment. To determine whether BAE is indicated, chest computed tomography (CT) is a useful noninvasive imaging modality for initial assessment of hemoptysis, determination of its etiology and origin, and affirmation of ground glass opacity suggesting hemorrhage and aspiration of blood. Although fiberoptic bronchoscopy (FOB) is essential for pathological examination, the efficacy of CT for etiology determination and patient evaluation is well established [6-8], and the combined use of high-resolution CT and FOB as complementary modalities increases the positive yield $[9,10]$.

Multidetector row computed tomography (MDCT) is useful for visualizing BAs and detecting ectopic origins, particularly when the patient is aged and has a tortuous aorta and/or extensive arteriosclerosis [11, 12]. Contrast material-enhanced MDCT and the created 3-dimensional images reportedly provide a more precise depiction of these vessels than does conventional bronchial arteriography (BAG). MDCT has thus emerged as a potential diagnostic tool for depicting and evaluating the systemic circulation and the trajectory of BAs and non-bronchial systemic arteries (NBSAs) causing hemoptysis [13-16]. The aim of the present study was to determine whether MDCT could be useful for identifying the anatomical characteristics of bleeding-related BAs, and for determining whether BAE is indicated or whether conservative treatment is sufficient.

\section{Materials and Methods}

\section{Patient Population}

In this retrospective investigation, we studied 41 consecutive patients [22 males and 19 females; age $64.5 \pm 12.0$ (mean \pm SD), range 25-80 years] referred to our institution with hemosputum and hemoptysis between June 2004 and May 2008. There they underwent contrast material-enhanced chest MDCT as part of the pre-therapeutic evaluation. This study was approved by our institutional review board and all patients were informed that the radiologic examination was primarily for clinical diagnosis and secondarily for radiological research, and all provided written consent. BAG was never performed for diagnostic purposes only. Patients presenting with massive hemoptysis ( $>500$ ml blood during the $24 \mathrm{~h}$ prior to treatment) requiring emergency BAE without contrast material-enhanced MDCT were excluded. Patients presenting with persistent hemoptysis that was resistant to conservative treatment were treated with BAG and BAE. Treatment decisions were made after discussion among the attending physicians and interventional radiologists.

We classified the patients and BAs into groups according to their BAE and bleeding status. Patients were classified according to whether or not they were treated with BAE, while the BAs were classified according to whether or not they were subjected to the
BAE procedure and whether or not they were the source of the bleeding. We evaluated the BAs in terms of their diameter and vascularization on MDCT.

Contrast Material and Scan Protocols for CT Examination

A 16-slice multidetector row CT scanner (Light Speed 16; GE Medical Systems, Milwaukee, Wisc., USA) with a detector configuration of $16 \times 0.625$ or $1.25 \mathrm{~mm}$ was used at $120 \mathrm{kVp}$ with an auto-adjusting mAs system (Auto mA; GE Medical Systems). The scan range was from the apex of the lung to its base. To identify the cause of bleeding and the affected side, scans were initially carried out without contrast material and with $100 \mathrm{ml}$ of nonionic contrast material containing $370 \mathrm{mg}$ iodine/ $\mathrm{ml}$ (Iopamilon 370; Bayer, Leverkusen, Germany) administered at a rate of 4 or $5 \mathrm{ml} / \mathrm{s}$ into an antecubital vein to add information about vascularization. This was followed by infusion of $20 \mathrm{ml}$ saline. Scans were begun $5 \mathrm{~s}$ after the bolus-tracking program detected an increase in 50 Hounsfield units (HU) in the CT value in the aorta.

\section{Hemorrhage Localization}

Although not all bronchial bleeding occurs from enlarged BAs, these arteries were considered responsible for hemoptysis if BAG and MDCT findings revealed the vessels to be enlarged, tortuous and supplying an area of lung parenchymal shadow, including ground glass opacity, condensation, neovascularity, hypervascularity, oozing, shunting into the pulmonary artery or vein, or the rarely seen BA aneurysm or extravasation of contrast medium [17]. Definitive determination of the hemorrhage site was based on BAG or MDCT that were in agreement with FOB findings of hemorrhage from the end of a bronchus including diseasespecific abnormalities. In patients who did not undergo FOB or FOB was normal or showed bilateral nonspecific abnormalities, when an infiltrative shadow in the pulmonary parenchyma and findings of angiography detected by MDCT were identical, they were also considered to be associated with bleeding and its etiology. Cases in which there was a discrepancy that prevented diagnosis of the bleeding site based on MDCT or FOB findings were defined as bleeding site unknown. We diagnosed the definitive or probable BAs as responsible for bleeding.

\section{Bleeding Status}

Each patient and BA were assigned bleeding status. They were classified as none, $<100 \mathrm{ml} / 24 \mathrm{~h}$, or $100-500 \mathrm{ml} / 24 \mathrm{~h}$. In cases in which we could not predict the bleeding sites, bilateral BAs were given the same bleeding status. If the bleeding sites were predictable, the contralateral BAs unrelated to the hemoptysis were given a status of none.

\section{Measurement of BA Diameter and Evaluation}

MDCT images were evaluated by 2 radiologists blinded to the patients' clinical information. The site of origin, distribution and findings were analyzed on transverse CT scans, maximum intensity projections and volume-rendered images on monitor slices of $0.625 \mathrm{~mm}$. The CT value (HU) of the aortic arch was measured. BA diameters were measured in the area of origin, at the level of the bronchial bifurcation in the mediastinum, and at the pulmonary hilum. The intercostobronchial artery (ICBA), which often has a branch designated as the origin of the right BA, was measured at a proximal site where the BA branches away from the intercostal artery trunk [18]. 

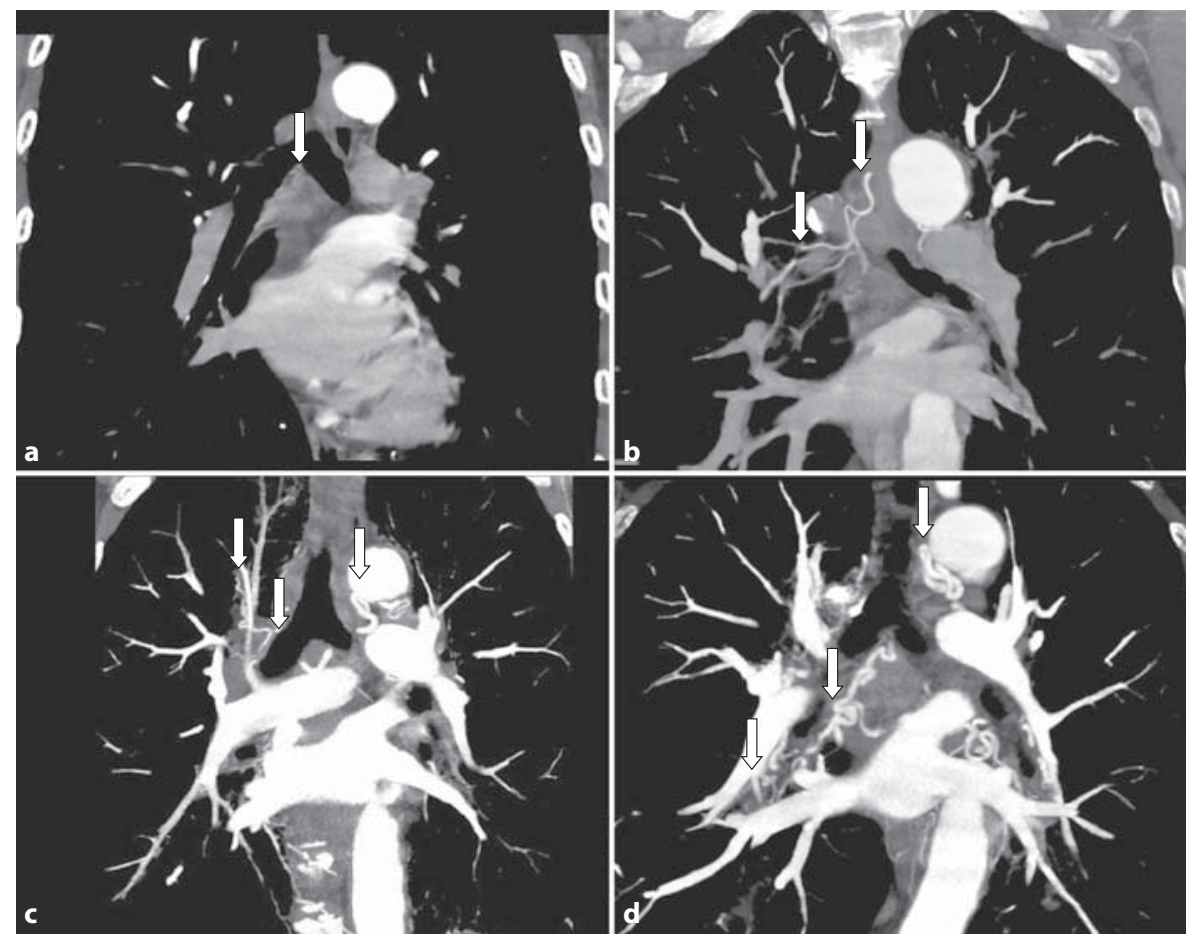

Fig. 1. MDCT scoring. BAs were visualized on MDCT (arrows) using maximum intensity projection (MIP) methods. a MDCT score of 0 (no findings): assigned BAs were narrowing and could not be traced in the mediastinal area. The image is from a 56 -yearold woman with trivial hemoptysis and considered within the normal limit. b Image with an MDCT score of 1: the BA is traceable to the pulmonary hilum and slightly dilated. The image is from a 74-year-old man with chronic bronchitis and who smokes. c Image with an MDCT score of 2: the BA shows moderate dilata- tion visible beyond the pulmonary hilum with some tortuosity. The image is from a 64-year-old woman with pulmonary emphysema and fibrosis. d Image with an MDCT score of 3: the BA shows bilateral maximal dilatation, and the area of vasodilatation clearly extends to the pulmonary parenchyma. Note that the BAs originate from the aorta and take a tortuous mediastinal course. The image is from a 60-year-old woman with recurrent moderate hemoptysis originating from right middle lobe bronchiectasia.
In patients receiving BAG, the bleeding site and vessel diameter were determined. BA diameters obtained during BAG were measured using digital angiography with the catheter diameter serving as a calibration standard and converted based on the number of pixels.

\section{Quantitative Image Analysis}

The radiologists used the following 4-point scale to evaluate BAs on MDCT images in terms of the degree of dilatation, tortuosity, and the detectability of the peripheral branches: $0=$ no findings, assigned BAs were narrowing and could not be traced in the mediastinal area (fig. 1a); 1 = traceable up to the level of the pulmonary hilum with/without slight dilatation (fig. 1b); 2 = moderate dilatation traceable beyond the pulmonary hilum and with some morphological abnormal findings (fig. 1c), and $3=$ maximal dilatation, tortuous and peripheral vasodilatation clearly traceable to the pulmonary parenchyma (fig. 1d).

\section{Statistical Analysis}

All statistical analyses were performed using StatView for Windows Version 5.0 (SAS Institute Inc., Cary, N.C., USA). Com- parisons of continuous variables between groups were made using unpaired Student's t tests. Comparisons among 3 groups were made using one-way analysis of variance followed by a post-hoc Fisher test. Categorical variables were compared using $\chi^{2}$ or Mann-Whitney $U$ tests. Values of $\mathrm{p}<0.05$ were considered significant.

\section{Results}

\section{Patient Characteristics}

The clinical characteristics of the patients, the causes of hemoptysis, and the BAE status are shown in table 1. Thirteen (32\%) patients had a history of smoking, and 2 (5\%) had used anticoagulant drugs. There was no significant demographic difference between the patients who received $\mathrm{BAE}$ and those who did not. To control hemorrhage (search and treatment), 8 patients received BAG; of 
Table 1. Patients characteristics and causes of hemoptysis

\begin{tabular}{|c|c|c|c|}
\hline Characteristic & $\begin{array}{l}\text { BAE } \\
\text { performed } \\
(\mathrm{n}=7)\end{array}$ & $\begin{array}{l}\text { BAE not } \\
\text { performed } \\
(\mathrm{n}=34)\end{array}$ & $\begin{array}{l}\mathrm{p} \\
\text { value }\end{array}$ \\
\hline Gender, male/female & $4 / 3$ & $18 / 16$ & 0.68 \\
\hline Age, years, mean $\pm S D$ & $63.6 \pm 18.0$ & $67.0 \pm 9.7$ & 0.47 \\
\hline \multicolumn{4}{|l|}{ Amount of hemoptysis, } \\
\hline$<100 / 100-500 \mathrm{ml}$ & $3 / 4$ & $23 / 11$ & 0.39 \\
\hline Hemoptysis-related deaths & 1 & 0 & 0.39 \\
\hline \multicolumn{4}{|l|}{ Causes of hemoptysis } \\
\hline Bronchiectasia & 1 & 7 & \\
\hline Chronic bronchitis $^{1}$ & 2 & 15 & \\
\hline Pulmonary emphysema & 1 & 4 & \\
\hline Primary lung cancer & 1 & 3 & \\
\hline Tuberculosis & 1 & 1 & \\
\hline Aspergilloma & 1 & 1 & \\
\hline Lung fibrosis & 0 & 2 & \\
\hline Cryptogenic & 1 & 5 & \\
\hline
\end{tabular}

Data are presented as numbers (n) and means \pm SD. BAE $=$ Bronchial artery embolization. The causes of hemoptysis include repetition in an underlying disease.

${ }^{1}$ Chronic bronchitis includes diffuse panbronchiolitis.

those, 7 received BAE in a total of 9 regions. Among the patients who received $\mathrm{BAE}$, the bleeding status of 3 was $<100 \mathrm{ml} / 24 \mathrm{~h}$; the other 4 showed $100-500 \mathrm{ml} / 24 \mathrm{~h}$. One patient died while still hospitalized. The cause of death was recurrent massive hemoptysis on day 2 after the first BAE. There were no hemoptysis-related deaths among the patients who received only conservative treatment.

\section{Characteristics of BAs Visualized by MDCT}

Table 2 summarizes the numbers of right and left BAs, their diameters, heights of their ostia, and bleeding sites. A total of 102 ostia and 96 traceable BAs were identified. Twelve patients had a branch from the ICBA, and 19 had 1 right $\mathrm{BA}$, while 14 had 2 right $\mathrm{BAs}$ that could be adequately evaluated. Twenty-five patients had 1 left BA, and 9 had 2 left BAs. These included 13 patients who had BAs arising from the descending aorta as a common trunk. Of those, 3 had a unilateral BA ostium that could not be depicted, and the anatomical structures were indistinct. The site of the ostium of the BA is usually considered orthotopic when the artery originates from the descending aorta, between the levels of the 5th and 6th thoracic vertebrae; all other heights are considered ectopic [14]. All arteries that underwent BAE were clearly visualized by MDCT. Seven arteries were located in orthotopic ostia
Table 2. Summary of BA findings on MDCT: anatomical characteristics and BAE/bleeding status

\begin{tabular}{|c|c|c|c|}
\hline & \multicolumn{2}{|l|}{ Location } & \multirow{2}{*}{$\begin{array}{l}\mathrm{p} \\
\text { value }\end{array}$} \\
\hline & right & left & \\
\hline \multicolumn{4}{|l|}{ Number of subjects } \\
\hline Ostium & 55 & 47 & \\
\hline Bifurcation & 49 & 39 & \\
\hline Hilar level & 37 & 30 & \\
\hline \multicolumn{4}{|l|}{ Height of ostium } \\
\hline Th5 & 18 & 14 & \\
\hline Th6 & 25 & 23 & \\
\hline Ectopic & 12 & 10 & \\
\hline BAE status $[\mathrm{BAE}(+) /(-)]$ & $5 / 50$ & $4 / 43$ & 0.99 \\
\hline Bleeding status [bleeding $(+) /(-)]$ & $15 / 40$ & $14 / 33$ & 0.83 \\
\hline \multicolumn{4}{|l|}{ Bleeding site, $\mathrm{n}$} \\
\hline Upper lobe & 3 & 6 & \\
\hline Middle lobe & 4 & 2 & \\
\hline Lower lobe & 3 & 5 & \\
\hline Diameter of ostium, mm & $2.6 \pm 0.8^{*}$ & $2.2 \pm 0.6$ & 0.004 \\
\hline \multicolumn{4}{|l|}{ Diameter at bronchial } \\
\hline bifurcation, mm & $2.3 \pm 0.8$ & $2.1 \pm 0.6$ & 0.33 \\
\hline Diameter at hilum, mm & $2.0 \pm 0.8$ & $2.0 \pm 0.7$ & 0.62 \\
\hline
\end{tabular}

Data are presented as numbers (n) and means $\pm \mathrm{SD} . \mathrm{BA}=$ Bronchial artery; $\mathrm{MDCT}=$ multidetector row computed tomography; $\mathrm{BAE}=$ bronchial artery embolization; $\mathrm{BAE}(+) /(-)=$ arteries receiving $(+)$ or not receiving $(-)$ BAE; bleeding $(+) /(-)=$ bronchial artery diagnosed as a source of bleeding $(+)$ or not a source of bleeding (-).

${ }^{*} \mathrm{p}<0.05$ vs. left bronchial artery.

and 2 arteries in ectopic ostia. Among all 41 patients, 13 (32\%) had at least $1 \mathrm{BA}$ of ectopic origin, and a total of 22 ectopic BAs were depicted. Among the detectable BAs, 6 were suboptimal with respect to the measurement of their diameter and were excluded from further analysis. At their origins, the diameters of right BAs were significantly larger than the left BAs $(\mathrm{p}=0.004)$.

\section{Bleeding Site}

Based on MDCT and FOB findings, the bleeding site was confirmed in 13 (32\%) patients. MDCT angiography and lung field findings were consistent in 12 (29\%) patients and the bleeding site was defined as probable. In 11 of these patients, hemoptysis originated from the right side; in 14 it originated from the left side. There were 16 (39\%) patients in whom the bleeding site was unknown. FOB was performed in 30 (73\%) patients. Abnormalities considered to be associated with the bleeding were detected in 20 patients ( 2 laterality and 18 lobes) and bilat- 
Table 3. Relation between BA diameters and the BAE/bleeding status

\begin{tabular}{llll}
\hline & $\begin{array}{l}\mathrm{BAE}(+) / \\
\text { bleeding }(+) \\
(\mathrm{n}=9)\end{array}$ & $\begin{array}{l}\mathrm{BAE}(-) / \\
\text { bleeding }(+) \\
(\mathrm{n}=20)\end{array}$ & $\begin{array}{l}\mathrm{BAE}(-) / \\
\text { bleeding }(-) \\
(\mathrm{n}=67)\end{array}$ \\
\hline $\begin{array}{l}\text { Diameter at ostium, mm } \\
\begin{array}{c}\text { Diameter at bronchial } \\
\text { bifurcation, mm }\end{array}\end{array}$ & $3.2 \pm 1.2^{\mathrm{a}}$ & $2.7 \pm 0.7^{\mathrm{a}}$ & $2.2 \pm 0.6$ \\
$\begin{array}{l}\text { Diameter at hilum, mm } \\
\text { Diame }\end{array}$ & $3.0 \pm 1.2^{\mathrm{a}, \mathrm{b}} \mathrm{b}$ & $2.4 \pm 0.6^{\mathrm{a}}$ & $2.0 \pm 0.6$ \\
\hline
\end{tabular}

Data are presented as means $\pm \mathrm{SD} . \mathrm{BA}=$ Bronchial artery; $\mathrm{BAE}=$ bronchial artery embolization; $\mathrm{BAE}(+) /$ bleeding $(+)=$ ar teries diagnosed a source of bleeding and receiving BAE; $\mathrm{BAE}(-) /$ bleeding $(+)=$ arteries diagnosed as a source of bleeding but not receiving $\mathrm{BAE}$; $\mathrm{BAE}(-) /$ bleeding $(-)=$ arteries diagnosed as not a source of bleeding and not receiving $\mathrm{BAE}$.

${ }^{\mathrm{a}} \mathrm{p}<0.05$ vs. BAE $(-) /$ bleeding $(-)$.

${ }^{\mathrm{b}} \mathrm{p}<0.05$ vs. BAE $(-) /$ bleeding $(+)$.

eral nonspecific abnormality in 3; the FOB results were normal in the remaining 7 patients. We detected lung cancer in 2 cases and bronchial erosion by metastatic lymph node invasion in 1 case.

\section{Comparison MDCT and BAG}

When we compared the diameters of BAs at 3 points (ostium, bronchial bifurcation and pulmonary hilum) using both BAG and MDCT, we obtained a good correlation between diameters estimated using the two techniques $(\mathrm{r}=0.709, \mathrm{p}<0.001$; fig. 2$)$.

\section{$B A$ Diameters on MDCT according to BAE/Bleeding}

Status

The diameters of BAs according to whether or not they bled [bleeding $(+)$ or bleeding $(-)$ ] and received $\mathrm{BAE}$ $[\mathrm{BAE}(+)$ or $\mathrm{BAE}(-)]$ are summarized in table 3 . The average diameters in each segment of BAs in the BAE $(+) /$ bleeding $(+)$ group were significantly larger than those in the $2 \mathrm{BAE}(-)$ groups. Moreover, the diameters of the $\mathrm{BAE}(+) /$ bleeding $(+)$ arteries remained largely unchanged from their origin through the mediastinum. By contrast, the diameters of $\mathrm{BAE}(-)$ arteries were smaller at the hilum and bronchial bifurcation than at their origin, whether or not the artery was the source of the bleeding. The sensitivity in the detection of the arteries at the level of the pulmonary hilum compared to the number of its ostium was high in both the $\mathrm{BAE}(+)(88 \%)$ and $\mathrm{BAE}(-)$ $(66 \% ; p=0.26)$ groups. The only exception among the $\mathrm{BAE}(+)$ group was a dilated $\mathrm{BA}$ that originated from the

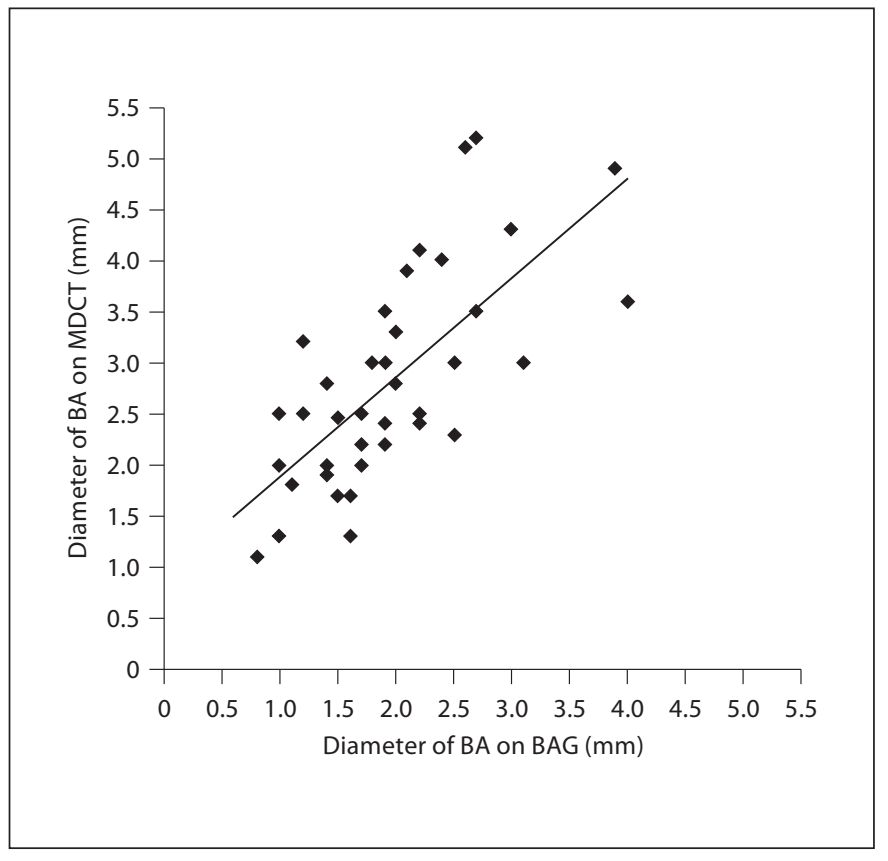

Fig. 2. Relationship between bronchial artery diameters on BAG and MDCT. Data are presented as means $\pm \mathrm{SD}$. BA $=$ Bronchial artery; $\mathrm{MDCT}=$ multidetector row computed tomography; $\mathrm{BAG}=$ bronchial artery angiography. $\mathrm{X}=\mathrm{BAG}$ diameter; $\mathrm{Y}=$ MDCT diameter. $Y=0.9718 X+0.9036 ; r=0.709, p<0.001$. Note the strong correlation between BAG and MDCT.

left subclavian artery and projected directly to the ipsilateral lung parenchyma with no detectable hilar path.

BAs defined as bleeding $(+)$ or considered to be related to the bleeding based on MDCT and/or FOB findings were significantly larger $(\mathrm{p}<0.05)$ at the origin, bronchial bifurcation and pulmonary hilum than arteries defined as bleeding(-). The sensitivity in the detection of bleeding $(+)$ arteries (96\%) at the level of the hilum was significantly greater than that of bleeding(-) arteries $(56 \% ; \mathrm{p}<0.001)$. Among bleeding $(+)$ arteries, there was no significant difference in diameter at the origin $(\mathrm{p}=$ 0.13 ) between those in the $\mathrm{BAE}(+)$ and $\mathrm{BAE}(-)$ groups, though a significant difference was seen at the bronchial bifurcation $(\mathrm{p}=0.049)$ and pulmonary hilum $(\mathrm{p}=0.03)$.

\section{Relationships between MDCT Score and BAE Status}

The relationship between MDCT score and the BAE status for each BA is shown in figure 3. Among the BAE $(+)$ group, there were 5 arteries with a score of 2 , and 4 with a score of 3 . In the BAE(-) group, there were 32 arteries with a score of 0,22 with a score of 1,15 with a score of 2 , and 18 with a score of 3 . No BAs with MDCT scores of 


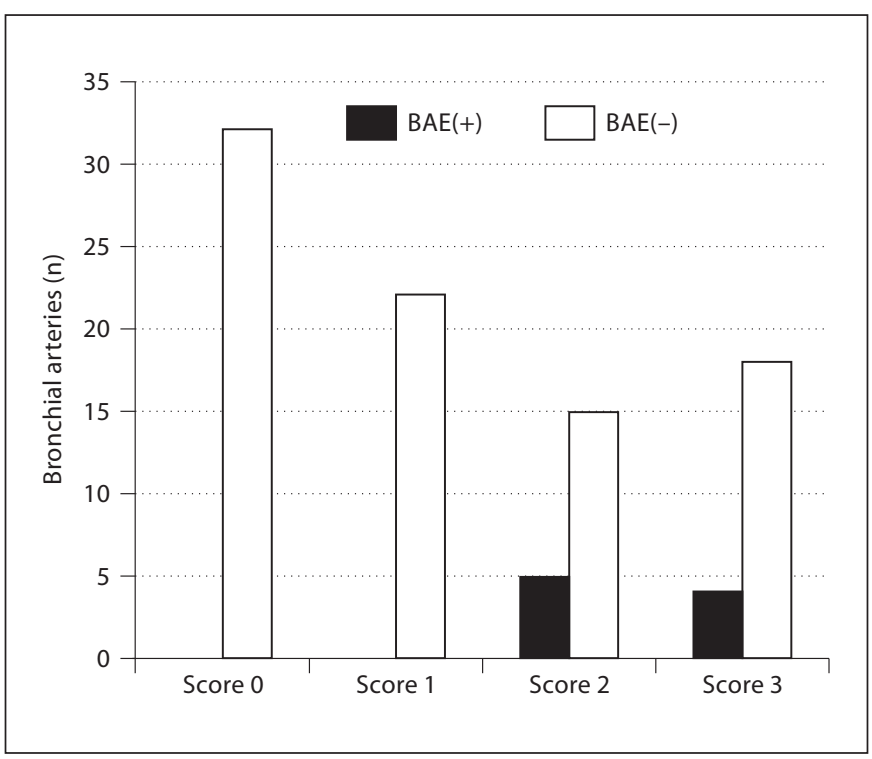

Fig. 3. Relationship between MDCT score and BAE status. MDCT = Multidetector row computed tomography; BAE = bronchial artery embolization; $\mathrm{BAE}(+)=$ arteries receiving $\mathrm{BAE}$; $\mathrm{BAE}(-)=$ arteries not receiving $\mathrm{BAE}$.

0 or 1 were administered $\mathrm{BAE}$, and the probability that BAE would be required declined significantly with lower MDCT scores $(\mathrm{p}=0.004)$. In multiple logistic regression analysis, ostium diameter and the amount of expectorated blood were independent predictive factors for BAE.

\section{Discussion}

In over $90 \%$ of hemoptysis cases requiring intervention with BAE or surgery, a $\mathrm{BA}$ is responsible for the bleeding [17]. BAE is mainly performed as an initial treatment, particularly in high-risk patients, because it is safer and less invasive than emergency surgery [3, 19-21], but the decision as to which BA should be embolized must take into account the possible necessity of an invasive $\mathrm{BAG}$ procedure. BA anatomy is highly variable and shows a great deal of individual difference; indeed, ectopic BAs can originate from the lower thoracic aorta, subclavian arteries, thyrocervical trunk, costocervical trunk, brachiocephalic artery, internal mammary artery, pericardiophrenic artery or inferior phrenic artery. In cases of hemoptysis, ectopic BAs, including NBSAs and the hard tortuous aortas seen in older patients, sometimes make catheterization, and thus conventional angi- ography, difficult. In many cases, this difficulty can be overcome with MDCT, as detection of BAs on MDCT is in good agreement with conventional angiographic findings in $86 \%$ of cases [15]. In addition, MDCT enables detection of $36 \%$ of ectopic origins and provides key information useful when making decisions about the management of hemoptysis [12]. Similarly, we detected ectopic BAs in 32\% patients in the present study.

Our findings indicated that BAs categorized as bleeding $[\mathrm{BAE}(+) /$ bleeding $(+)$ or $\mathrm{BAE}(-) /$ bleeding $(+)]$ were significantly larger than those categorized as not bleeding $[\mathrm{BAE}(-) /$ bleeding(-)]. BAs receiving BAE had larger diameters that did not change from the ostium of the artery through the mediastinum to the hilum. We also found that there is a good correlation between the BA diameters visualized on BAG and MDCT, though estimates tended to be larger with MDCT than with BAG. Earlier reports suggest that most BAs of $>2 \mathrm{~mm}$ in diameter on conventional contrast CT should be considered abnormal and dilated $[17,22]$. Based on autopsies of adult human lungs, one would expect normal BA diameters to be approximately $1.5 \mathrm{~mm}$ at the hilum and decline to $0.5-0.75 \mathrm{~mm}$ at the point of entry into the lung parenchyma [23]. Our investigation showed that, for BAs other than those in the $\mathrm{BAE}(+) /$ bleeding $(+)$ group, diameters at the level of the pulmonary hilum were significantly smaller than at the ostium, and that diameters tended to become smaller as one measured more peripherally. At the hilum, diameters of BAE(-)/bleeding(-) arteries averaged $1.8 \pm 0.6 \mathrm{~mm}$ on MDCT, which is larger than previously reported. But considering that our investigation was limited to patients presenting with hemoptysis and included no healthy individuals, this would seem a reasonable estimate. Our estimates of BA diameter on MDCT would also be affected by a vessel's trajectory, its volume, the surrounding tissues, and the scanning technique [24]. In addition, BAs too small to detect would be excluded from our analysis; consequently, we may overestimate the diameters of BAs not responsible for hemorrhage. Yoon et al. [14] reported that BAs responsible for bleeding can frequently be visualized and traced from their origin to the level of the pulmonary hilum (74\%), whereas only $11 \%$ of BAs not responsible for hemorrhage can be traced that far. In our investigation, we were able to visualize $96 \%$ of BAs responsible for bleeding and $56 \%$ of those not responsible for bleeding. Our greater ability to visualize the arteries, regardless of their bleeding status, is likely the result of our administration of high-density contrast material. Following injection of the contrast medium, the CT number in the descending aorta was $463 \pm 84 \mathrm{HU}$, which is 
sufficient to visualize arteries smaller than the previously reported hepatic and coronary arteries $[25,26]$.

BAs with low MDCT scores were significantly less likely to require BAE than those with high MDCT scores. And in multiple logistic regression analysis, it was found that detection of a larger ostial diameter and the bleeding status, whether or not the BA under study was a source of the hemoptysis, affected the radiologists' decision as to which BA should be embolized. Although the BA that is the source of the hemoptysis may not always have a large diameter, its diameter and peripheral traceability are important reciprocal factors contributing to the MDCT score. BAs that did not pass though the mediastinum were not suitable for our quantitative image analysis, however. Moreover, the amount of hemoptysis is not equal to that seen in the lower respiratory tract; consequently, the amount of bleeding may be underestimated. We therefore suggest that it is also important to take the patient's cardiorespiratory status and risk of asphyxiation into account, since BAE could prevent development of the next life-threatening hemoptysis. That said, because we had only one hemoptysis-related death, our findings do not clarify whether BAE changed the outcomes of patients in the $\mathrm{BAE}(+)$ group. In an area of high tuberculosis incidence, risk factors for recurrence of hemoptysis and its management were reported [27,28]. It is inevitable to perform BAG to detect fine bleeding vessels in persistent hemoptysis patients, even when MDCT showed no findings, because spatial resolution of MDCT is inferior to that of BAG. Although fine bleeding from BA can be detected only by BAG, we can notice diagnostic signs of bleeding arteries that usually indicate consideration of BAE when significant tortuosity and vasodilatation [29] were detected by MDCT. The fact that some hemoptysis patients recovered with only conservative treatment suggests that the relationships between diameter, quantity of hemoptysis, MDCT score, and the need for BAE remain unclear.

In summary, by using contrast material-enhanced MDCT, we were able to identify individual anatomical variations, including ectopic origins and probable bleeding-related BAs, as well as bilateral small BA diameters with low MDCT scores not requiring BAE, in patients not presenting with massive hemoptysis. From a practical standpoint, BA evaluation with MDCT could be useful for determining appropriate initial management of hemoptysis; that is, whether conservative treatment is sufficient or whether a patient should be readied for emergency BAE.

\section{References}

1 Fraser RS, Pare P, Pare PD: Hemoptysis; in Fraser RS, Pare P, Pare PD (eds): Diseases of the Chest. Philadelphia, Saunders, 1988, pp 394-396.

-2 Endo S, Otani S, Saito N, Hasegawa T, Kanai Y, Sato Y, Sohara Y: Management of massive hemoptysis in a thoracic surgical unit. Ann Thorac Surg 2002;74:185-190.

-3 Fartoukh M, Khalil A, Louis L, Carette MF, Bazelly B, Cadranel J, Mayaud C, Parrot A: An integrated approach to diagnosis and management of severe haemoptysis in patients admitted to the intensive care unit: a case series from a referral centre. Respir Res 2007;8:11-20.

4 Shigemura N, Wan IY, Yu SC, Wong RH, Hsin MK, Thung HK, Lee TW, Wan S, Underwood MJ, Yim AP: Multidisciplinary management of life-threatening massive hemoptysis: a 10-year experience. Ann Thorac Surg 2009;87:849-853.

$\checkmark 5$ Mal H, Rullon I, Mellot F, Brugière O, Sleiman C, Menu Y, Fournier M: Immediate and long-term results of bronchial artery embolization for life-threatening hemoptysis. Chest 1999;115:996-1001.
6 Naidich DP, Funt S, Ettenger NA, Arranda C: Hemoptysis: CT-bronchoscopic correlations in 58 cases. Radiology 1990;177:357-362.

-7 McGuinness G, Beacher JR, Harkin TJ, Garay SM, Rom WN, Naidich DP: Hemoptysis: prospective high-resolution CT/bronchoscopic correlation. Chest 1994;105:11551162 .

$\checkmark 8$ Revel MP, Fournier LS, Hennebicque AS, Cuenod CA, Meyer G, Reynaud P, Frija G: Can CT replace bronchoscopy in the detection of the site and cause of bleeding in patients with large or massive hemoptysis? AJR Am J Roentgenol 2002;179:1217-1224.

-9 Hirshberg B, Biran I, Glazer M, Kramer MR: Hemoptysis: etiology, evaluation, and outcome in a tertiary referral hospital. Chest 1997;112:440-444.

10 Tsoumakidou M, Chrysofakis G, Tsiligianni I, Maltezakis G, Siafakas NM, Tzanakis N: A prospective analysis of 184 hemoptysis cases: diagnostic impact of chest X-ray, computed tomography, bronchoscopy. Respiration 2006;73:808-814.
11 Khalil A, Fartoukh M, Tassart M, Parrot A, Marsault C, Carette MF: Role of MDCT in identification of the bleeding site and the vessels causing hemoptysis. AJR Am J Roentgenol 2007;188:W117-W125.

12 Hartmann IJ, Remy-Jardin M, Menchini L, Teisseire A, Khalil C, Remy J: Ectopic origin of bronchial arteries: assessment with multidetector helical CT angiography. Eur Radiol 2007;17:1943-1953.

${ }_{13}$ Yoon W, Kim YH, Kim JK, Kim YC, Park JG, Kang HK: Massive hemoptysis: prediction of nonbronchial systemic arterial supply with chest CT. Radiology 2003;227:232-238.

14 Yoon YC, Lee KS, Jeong YJ, Shin SW, Chung MJ, Kwon OJ: Hemoptysis bronchial and nonbronchial systemic arteries at 16-detector row CT. Radiology 2005;234:292-298.

15 Remy-Jardin M, Bouaziz N, Dumont P, Brillet PY, Bruzzi J, Remy J: Bronchial and nonbronchial systemic arteries at multi-detector row CT angiography: comparison with conventional angiography. Radiology 2004;233: 741-749. 
16 Chung MJ, Lee JH, Lee KS, Yoon YC, Kwon OJ, Kim TS: Bronchial and nonbronchial systemic arteries in patients with hemoptysis: depiction on MDCT angiography. AJR Am J Roentgenol 2006;186:649-655.

17 Yoon W, Kim JK, Kim YH, Chung TW, Kang HK: Bronchial and nonbronchial systemic artery embolization for life-threatening hemoptysis: a comprehensive review. RadioGraphics 2002;22:1395-1409.

18 Hasegawa I, Boiselle PM, Hatabu H: Bronchial artery dilatation on MDCT scans of patients with acute pulmonary embolism: comparison with chronic or recurrent pulmonary embolism. AJR Am J Roentgenol 2004;182:67-72.

-19 Fernando HC, Stein M, Benfield JR, Link DP: Role of bronchial artery embolization in the management of hemoptysis. Arch Surg 1998; 133:862-866.
20 Fartoukh M, Khalil A, Louis L, Carette MF, Bazelly B, Cadranel J, Mayaud C, Parrot A: An integrated approach to diagnosis and management of severe haemoptysis in patients admitted to the intensive care unit: a case series from a referral centre. Respir Res 2007;8:11.

21 Marshall TJ, Jackson JE: Vascular intervention in the thorax: bronchial artery embolization for hemoptysis. Eur Radiol 1997;7: 1221-1227.

22 Furuse M, Saito K, Kunieda E, Aihara T, Touei H, Ohara T, Fukushima K: Bronchial arteries: CT demonstration with arteriographic correlation. Radiology 1987;162: 393-398.

23 Pump KK: Distribution of bronchial arteries in the human lung. Chest 1972;62:447-452.

24 Kauczor HU, Schwickert HC, Mayer E, Schweden F, Schild HH, Thelen M: Spiral CT of bronchial arteries in chronic thromboembolism. J Comput Assist Tomogr 1994;18: 855-861.

-25 Tanikake M, Shimizu T, Narabayashi I, Matsuki M, Masuda K, Yamamoto K, Uesugi Y, Yoshikawa S: Three-dimensional CT angiography of the hepatic artery: use of multidetector row helical $\mathrm{CT}$ and a contrast agent. Radiology 2003;227:883-889.
6 Utsunomiya D, Awai K, Sakamoto T, Nishiharu T, Urata J, Taniguchi A, Nakaura T, Yamashita Y: Cardiac 16-MDCT for anatomic and functional analysis: assessment of a biphasic contrast injection protocol. AJR Am J Roentgenol 2006;187:638-644.

-27 van den Heuvel MM, Els Z, Koegelenberg CF, Naidu KM, Bolliger CT, Diacon AH: Risk factors for recurrence of haemoptysis following bronchial artery embolisation for life-threatening haemoptysis. Int J Tuberc Lung Dis 2007;11:909-914.

28 Gross AM, Diacon AH, van den Heuvel MM, van Rensburg J, Harris D, Bolliger CT: Management of life-threatening haemoptysis in an area of high tuberculosis incidence. Int J Tuberc Lung Dis 2009;13:875-880.

29 Swanson KL, Johnson CM, Prakash UB, McKusick MA, Andrews JC, Stanson AW: Bronchial artery embolization: experience with 54 patients. Chest 2002;121:789-795. 